\title{
Component Integration Effects in 4-junction Solar Cells with Dilute Nitride 1eV Subcell
}

\author{
I. García, M. Ochoa, I. Lombardero, L. Cifuentes, P. Caño, M. Hinojosa, I. Rey-Stolle and C. Algora \\ A. D. Johnson and J. I. Davies \\ K.H. Tan, W.K. Loke, S. Wicaksono and S. F. Yoon
}

\begin{abstract}
A GaInP/Ga(In)As/GaNAsSb/Ge 4-junction solar cell grown using combined MOVPE+MBE growth is used to analyze the effects during the integration of the subcell components into the full $\mathbf{4 J}$ structure. In this preliminary study, the Ge subcell is observed to suffer about $15 \% J_{s c}$ drop and $\sim 50 \mathrm{mV} \mathrm{V}_{\text {oc }}$ loss at 1-sun, while the $\mathrm{V}_{\text {oc }}$ of the GaNAsSb subcell drops by as much as $\sim 140 \mathrm{mV}$. The degradation of the Ge and GaNAsSb subcells in the current-matched $4 \mathrm{~J}$ structure can hinder its efficiency potential to a higher extent than in the $\mathrm{GaInP} / \mathrm{Ga}(\mathrm{In}) \mathrm{As} / \mathrm{Ge}$ 3J. Besides, high quality GaNAsSb and $\mathrm{Ge}$ subcells would still limit the current and require redesigning the top subcells to achieve optimum efficiencies.
\end{abstract}

\section{INTRODUCTION}

Monolithic and lattice-matched multijunction solar cells are attractive for fabrication simplicity and, hence, lower cost. Using dilute-nitride subcells is promising and has already shown high efficiencies in $3 \mathrm{~J}$ solar cells grown by MBE [1], and combined MOVPE + MBE [2]. A GaInP/Ga(In)As/ GaNAsSb/Ge $4 \mathrm{~J}$ solar cell, using the Ge substrate as an active subcell, conceptually consists on just adding the dilute nitride junction to the GaInP/Ga(In)As/Ge 3J solar cell. Currently, attaining a dilute-nitride subcell with longer minority carrier diffusion length so as not to limit the performance of the whole $4 \mathrm{~J}$ structure is the main limitation to achieving high efficiencies. Therefore, a high fraction of the work to do consists on further developing the semiconductor deposition technique. Ideally, MOVPE would be used to grow the full $4 \mathrm{~J}$ structure, given its advantages with respect to other growth techniques concerning high volume fabrication cost. However, attaining high quality dilute-nitride subcells by MOVPE has been elusive, although a recent publication has shown positive steps towards achieving this [3]. Conversely, better carrier diffusion lengths have been achieved by MBE, so as to obtain high efficiency $3 \mathrm{~J}$ solar cells [1]. Thus, the combined MOVPE + MBE method makes sense at this stage of development and should allow the attainment of high efficiencies in the short term.

The $4 \mathrm{~J}$ structure is complex and involves growing a large number of layers of different materials, growth temperatures and times. It is important to assess the integration of all these components to detect and take care of problems in advance, and facilitate the development of a high efficiency $4 \mathrm{~J}$ solar cell. The focus has to be put in identifying and quantifying the effects that put in risk the efficiency potential of this $4 \mathrm{~J}$ solar cell and redesigning, if possible, the growth process to minimize their impact.

In this work we first present our $4 \mathrm{~J}$ solar cell, based on a prototype structure achieved by the combined MOVPE + MBE method, and using a GaNAsSb junction. This solar cell exhibits a low performance due to the limiting behavior of the dilute nitride subcell, but represents a success in the implementation of the $4 \mathrm{~J}$ structure. However, this structure is used to analyze the integration of subcells into the full $4 \mathrm{~J}$ structure as the main point of this work. In this preliminary work, we focus on the GaNAsSb and, mostly, the Ge bottom subcell, which are subjected to long annealing times during the growth of the upper structures. It is found that significant loses are at stake, mainly in the Ge and GaNAsSb subcells, which can limit the potential of this $4 \mathrm{~J}$ solar cell structure to compete in efficiency with other architectures.

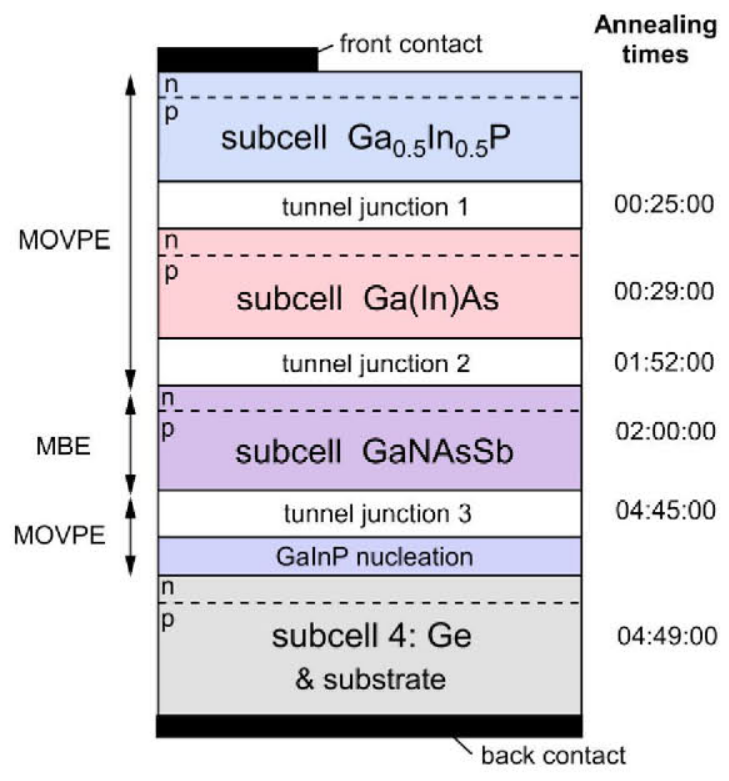

Fig. 1. Structure of the 4J solar cell presented, with indication of the parts grown by MOVPE and MBE, and the annealing times that each component subcell and tunnel junction suffer during the rest of the structure. 


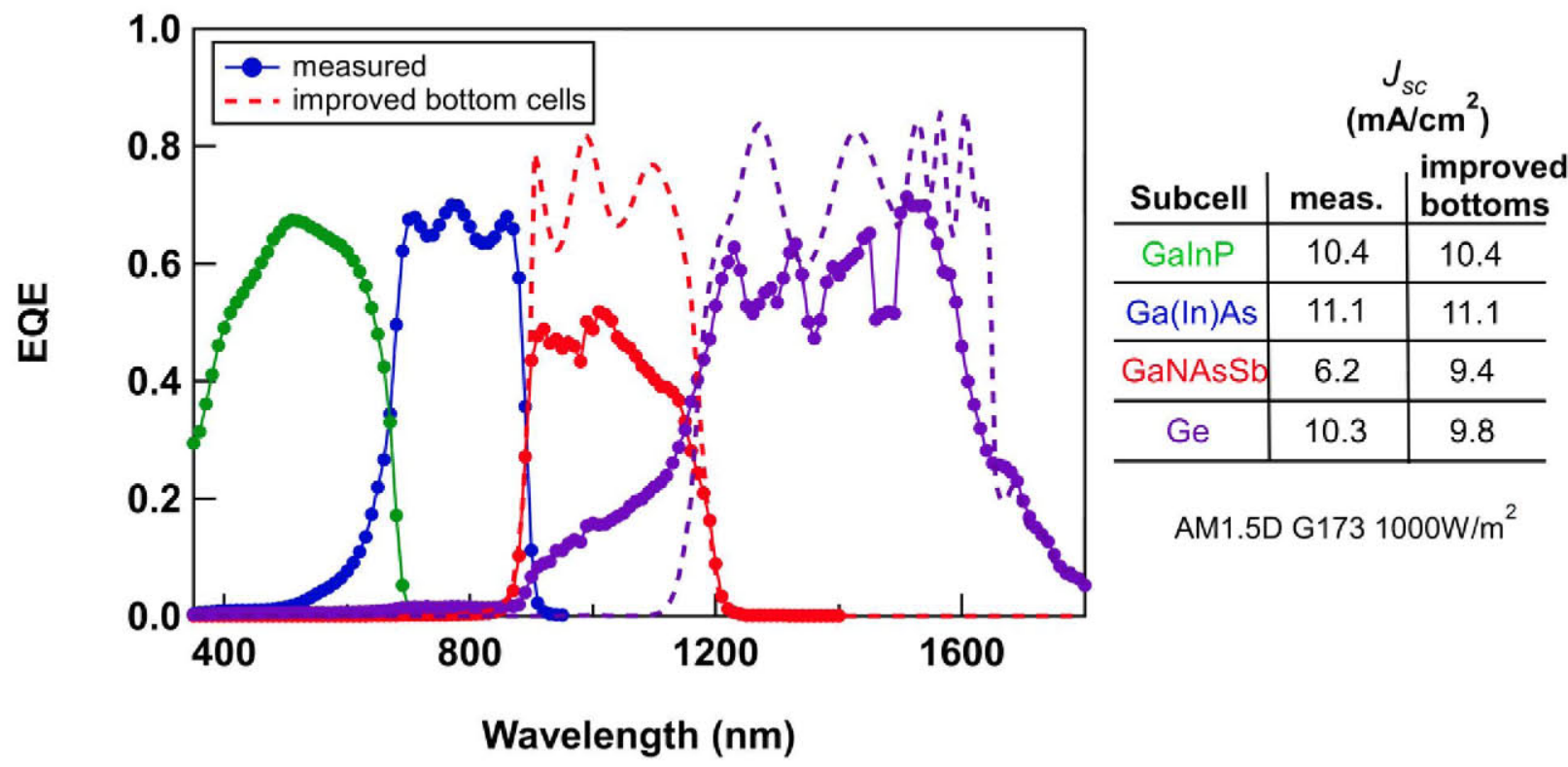

Fig. 2. Symbols: measured EQE of the 4J solar cell implemented; dashed line: modeled GaNAsSb and Ge subcells using minority carrier collection efficiencies of 1 and optically thick GaNAsSb. The table on the right shows the $\mathrm{J}_{\mathrm{sc}}$ obtained using these EQE and the AM1.5D G173 solar spectrum.

\section{MOVPE+MBE 4J SOLAR CELL}

The structure consists on a $\mathrm{GaInP} / \mathrm{Ga}$ (In)As/GaNAsSb/Ge structure grown lattice-matched to a Ge substrate, which is also the $4^{\text {th }}$ junction, as shown in Figure 1. The growth process comprises a sequence of MOVPE (GaInP nucleation on Ge and tunnel junction) + MBE (GaNAsSb subcell) + MOVPE ( $\mathrm{GaInP} / \mathrm{Ga}$ (In)As top junctions, including tunnel junctions). Given the low minority carrier diffusion length in the dilute nitride material, the GaNAsSb subcell absorber was made as thin as $1 \mu \mathrm{m} \mathrm{[4],} \mathrm{[5].} \mathrm{These} \mathrm{structures} \mathrm{were} \mathrm{processed} \mathrm{into}$ solar cell devices using gold-based metal and standard photolithography techniques. No anti-reflection coating has been applied to these solar cells for this study.

\section{A. External Quantum Efficiency (EQE).}

The measured external quantum efficiency (EQE) of this solar cell is shown in Figure 2, with the corresponding $\mathrm{J}_{\mathrm{sc}}$ calculated using the AM1.5D G173 solar spectrum detailed in the table on the right. The top subcells exhibit the usual EQE with good $\mathrm{J}_{\mathrm{sc}}$ values, while the low EQE of the nitride subcell limits the $\mathrm{J}_{\mathrm{sc}}$ of the whole device. The $\mathrm{J}_{\mathrm{sc}}$ of the Ge bottom junction is also low considering the fact that the light absorption in the GaNAsSb junction is incomplete and the Ge bottom cell has an unrealistic extended response in the short wavelength range. As a first assessment of the potential and loss sources in this EQE, the modeled EQE of improved GaNAsSb and Ge subcell was calculated. The Generalized
Matrix Method was used to compute the absorption in each layer [6] and the EQE was obtained applying a carrier collection efficiency to the absorber layers to fit the experimental EQE. Then, the improved EQE was calculated using an optically thick GaNAsSb subcell and a minority carrier collection efficiency of 1 in both subcells. The resulting EQEs are also shown in Figure 2. A strong effect on the GaNAsSb subcell EQE and $\mathrm{J}_{\mathrm{sc}}$ is observed, as expected, but, the Ge subcell carrier collection efficiency can also be improved to achieve a higher $J_{\text {sc. }}$. This can be observed as a higher average value of the EQE. Note that the $\mathrm{J}_{\mathrm{sc}}$ of the $\mathrm{Ge}$ subcell in the "improved bottoms" case is lower than the measured because the GaNAsSb subcell in this case is optically thick. The cause of the low EQE in this Ge subcell is discussed in Section III. Finally, according to the table on the right, the bottom subcells still limit the $\mathrm{J}_{\mathrm{sc}}$ of the $4 \mathrm{~J}$ even improving their carrier collection efficiency. This means that realizing the full efficiency potential of this $4 \mathrm{~J}$ solar cell will involve making the top cells more transparent by changing their thickness or, optimally, their bandgap.

\section{B. Open Circuit Voltage $\left(V_{o c}\right)$.}

Light I-V curves were measured on these $4 \mathrm{~J}$ solar cells. We have published previous work about the performance and particularities in the shape of the I-V curve of the $4 \mathrm{~J}$, predicted using simulations with inputs from the characterization results of GaNAsSb $1 \mathrm{~J}$ and GaNAsSb/Ge 2J cells [4], [5]. As for this work, we are mostly interested in the open circuit voltage $\left(V_{o c}\right)$, which we showed to be around $420 \mathrm{mV}$ for the 


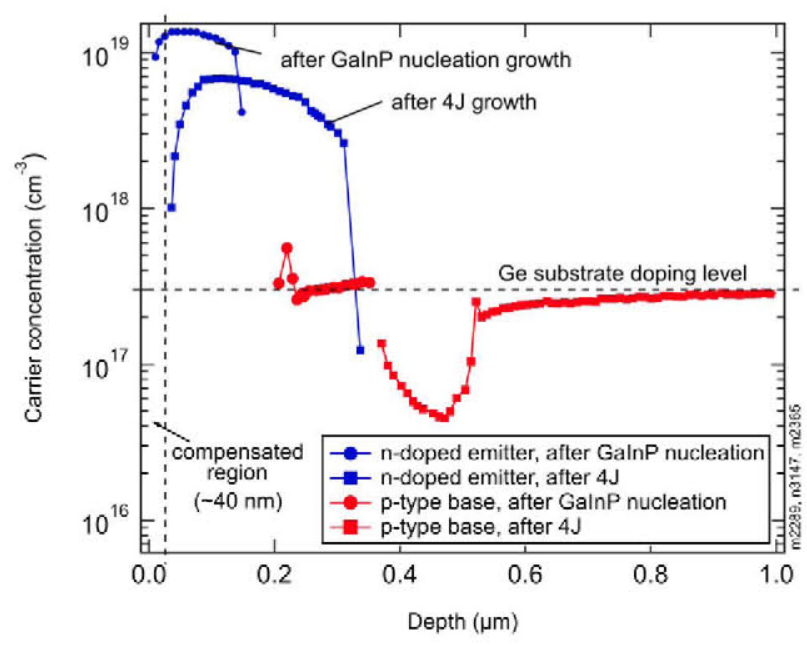

Fig. 3. Carrier concentration profiles of Ge subcells before and after growth of the full 4J structure.

GaNAsSb solar cell as an independent device [4]. The 4J solar cell presented here was measured under a calibrated solar simulator at NREL, and exhibited a $\mathrm{V}_{\mathrm{oc}}$ at 1-sun of $2.44 \mathrm{~V}$ and $2.48 \mathrm{~V}$ for the AM1.5d and AM0 solar spectra, respectively. This $V_{o c}$ is rather low, as compared to state-of-the-art GaInP/Ga(In)As/Ge 3J solar cells developed in our lab, which exhibit an $V_{o c}$ of around the same value [7]. This means that the $\mathrm{V}_{\mathrm{oc}}$ increase in the $4 \mathrm{~J}$ expected with the insertion of the GaNAsSb subcell does not occur, which eliminates any performance advantage with respect to the $3 \mathrm{~J}$ solar cell. Part of this voltage loss is due to degradation of the Ge and GaNAsSb subcells, as discussed in next section.

\section{COMPONENT INTEGRATION ANALYSIS}

In order to elucidate the origin of the voltage loss, and to assess the degradation of other possible solar cell performance parameters such as the EQE, an analysis of the possible sources of subcell degradation when integrating them into a $4 \mathrm{~J}$ solar cell was carried out. The first suspect in the list of possible causes is the thermal loads applied to the subcells. In Figure 1 we show a list of the annealing times to which each subcell of the $4 \mathrm{~J}$ structure is subjected to. The growth temperatures range from $550{ }^{\circ} \mathrm{C}$ for the tunnel junctions to $675^{\circ} \mathrm{C}$ for the subcell growth. The GaNAsSb subcell growth includes a $700{ }^{\circ} \mathrm{C}$ annealing step for 5 minutes to improve its properties, similarly as with other dilute-nitride materials [8][11]. The insertion of the dilute nitride subcell increases the thermal load to the Ge bottom subcell, as compared to the $3 \mathrm{~J}$ case. Considering the different growth times and temperatures used for each component in the $4 \mathrm{~J}$ structure, it is not obvious to predict the real impact of this added thermal load, and we assessed it empirically by examining samples at different stages of the MOVPE + MBE + MOVPE process.

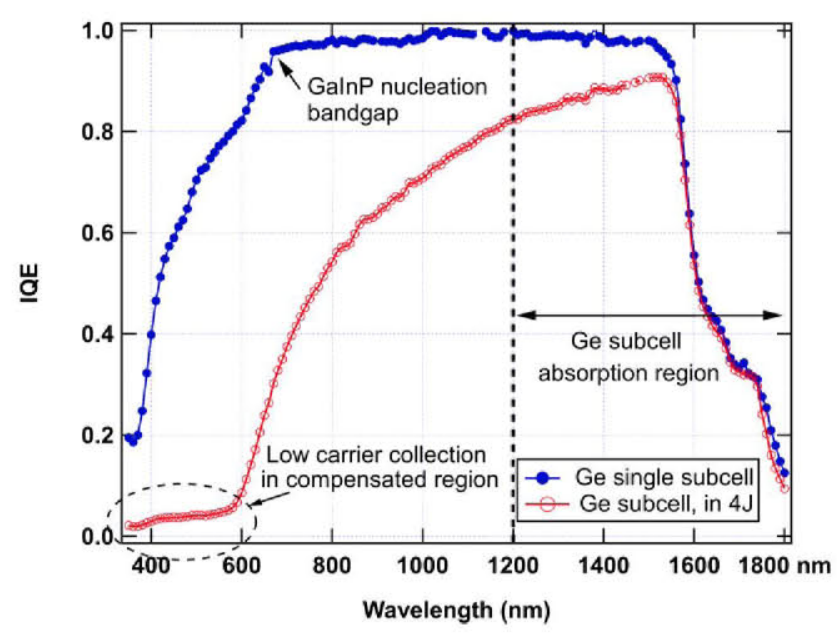

Fig. 4. IQE of the Ge subcell after being grown as a 1-junction, and in the 4-junction solar cell (measured after etching the top layers)

A first test was to measure the dopant diffusion in the $\mathrm{Ge}$ junction, which we did by taking electro-chemical capacitance-voltage (ECV) profiles of Ge junctions, shown in Figure 3. The profiles show the n-type and p-type carrier concentration corresponding to the emitter and base, respectively. The base carrier concentration corresponds to the Ge substrate nominal doping, around $3 \cdot 10^{17} \mathrm{~cm}^{-3}$. The emitter doping is achieved by diffusion of group- $\mathrm{V}$ elements (phosphorus) during the growth of the GaInP nucleation layer. Group-III elements also diffuse and give rise to a lower effective doping at the surface of the emitter. Preliminary SIMS measurements appear to indicate that this is caused mainly by indium diffusion.

The dopant diffusion at the emitter during the growth of the 4J structure is evident in Figure 3: the average carrier concentration level and thickness of the emitter changes substantially. Not less importantly, a strongly compensated region appears at the surface of the emitter, due to further indiffusion of group-III elements. The carrier concentration level rises sharply to achieve its peak value at a depth of around $100 \mathrm{~nm}$. The electric field created in this region can be expected to act as a sink for minority carriers which are lost by recombination.

IQE measurements were taken on these Ge subcells to assess this, as shown in Figure 4. Firstly, the IQE of the Ge subcell obtained right after the growth of the GaInP nucleation layer shows a value near 1 for all the wavelength range between the Ge and GaInP nucleation layer absorption edges. As for the Ge subcell after the growth of the $4 \mathrm{~J}$, an important degradation of the carrier collection efficiency in the lower wavelength region can be observed. The degraded $\mathrm{Ge}$ junction can be accurately modeled including the compensated layer $(\sim 40 \mathrm{~nm})$ with 0 collection efficiency, and a degraded carrier collection in the emitter bulk. 
It is important to point out that the apparent severity of the IQE degradation shown in Figure 4 is lessened by realizing the fact that, in the $4 \mathrm{~J}$, the Ge subcell absorption range falls in the higher photon wavelength region, where the degradation is not so severe. However, for the range of Ge subcell absorption in the $4 \mathrm{~J}$, the $\mathrm{J}_{\mathrm{sc}}$ drop due to the degraded IQE is as high as $\sim 15 \%$ calculated for the AM1.5D-G173 solar spectrum.

As expected, the $\mathrm{V}_{\text {oc }}$ of the Ge subcell exhibits changes too. The study of the degradation of the $V_{o c}$ in the subcells is complicated in these $4 \mathrm{~J}$ devices: the electroluminescence technique [12] cannot be used due to the undetectable emission of light from the dilute nitride subcell. In this work, the Ge subcell voltages were accessed by etching the upper layers. A drop of $\sim 50 \mathrm{mV}$ after the growth of the $4 \mathrm{~J}$ structure can be observed. We are currently carrying out experiments to elucidate the evolution of the $\mathrm{V}_{\mathrm{oc}}$ during the growth of the different $4 \mathrm{~J}$ structure components.

All in all, an important degradation in the Ge junction when integrating it in the $4 \mathrm{~J}$ structure is observed, which can limit the performance potential of this solar cell architecture. However, measures to minimize this degradation can be taken, such as redesigning the critical steps that determine the phosphorous and indium in-diffusion into the Ge to form the emitter during growth.

As for the GaNAsSb subcell, it is submitted to an intentional annealing in order to improve its material properties, as commented before. Additionally, the growth of the $\mathrm{GaInP} / \mathrm{Ga}(\mathrm{In})$ As top subcells involves an extra annealing of this subcell, of about 2 hours at $\sim 675^{\circ} \mathrm{C}$. However, no significant changes have been observed in its IQE, in the range of photon energies that it absorbs. However, the $V_{o c}$ of GaNAsSb single-junction solar cells drop by as much as $\sim 140 \mathrm{mV}$ at 1-sun. The intentional annealing used to improve the GaNAsSb subcell is normally designed concerning the temperature and time used, so that there exists an optimum combination of these parameters. The additional annealing of our GaNAsSb subcell appears to deviate the total thermal load from the optimum required. This means that the optimization of the intentional annealing will need to take into account the subsequent, unwanted annealing that the GaNAsSb subcell suffers during the growth of the $4 \mathrm{~J}$ structure.

\section{CONCLUSIONS}

We have presented a GaInP/Ga(In)As/GaNAsSb/Ge 4J solar cell grown using MOVPE+MBE combined method. The GaNAsSb subcell quality is not enough to allow a high performance $4 \mathrm{~J}$ solar cell, yet. However, this $4 \mathrm{~J}$ structure was used for a preliminary assessment of effects during the integration of the subcell components. The Ge subcell has been found to degrade significantly during the growth of the $4 \mathrm{~J}$ structure: its emitter carrier concentration profile changes substantially and this is correlated with a significant drop in the IQE (and $\mathrm{J}_{\mathrm{sc}}$ ) and $\mathrm{V}_{\mathrm{oc}}$. Moreover, the GaNAsSb subcell shows a large $V_{o c}$ drop, but no significant change in its IQE. Additional work to complete this analysis and extend it to the other subcells and tunnel junctions is underway. However, we can conclude that the efficiency potential of this $4 \mathrm{~J}$ architecture can be significantly lowered if the observed degradation of the Ge and GaNAsSb subcells is not minimized by a proper design of the semiconductor structure growth routine. An important remark is that, even in the case of achieving high quality GaNAsSb and Ge subcells, they would still limit the current in the $4 \mathrm{~J}$ and further optimization would require bandgap/thickness changes in the $\mathrm{GaInP}$ and $\mathrm{Ga}(\mathrm{In}) \mathrm{As}$ top junctions. Finally, the conclusions of this paper should be generalizable to other structures using dilute-nitride subcells, since the growth conditions used in our case are not harsher than commonly used.

\section{ACKNOWLEDGEMENTS}

This work has been supported by the European Commission by means of the LONGESST project (FP7 grant Agreement Number 607153), by the Spanish MINECO through the projects TEC2014-54260-C3-1-P and PCIN-2015-181-C02-02 and by the Comunidad de Madrid through the project MADRID-PV (S2013/MAE-2780). I. García is funded by the Spanish Programa Estatal de Promoción del Talento y su Empleabilidad through a Ramón y Cajal grant. I. Lombardero and M. Hinojosa are funded by the Spanish MINECO through an FPU14 and FPU15 grants, respectively. The authors want to acknowledge the technical support by J. Bautista. 1-sun I-V measurements with calibrated light spectrum were carried out at NREL, for which the authors are indebted to J. Geisz and D. Friedman.

\section{REFERENCES}

[1] R. Jones-Albertus et al., "Using Dilute Nitrides to Achieve Record Solar Cell Efficiencies" MRS Online Proc. Libr. Arch., vol. 1538, pp. 161-166, Jan. 2013.

[2] A. Tukiainen et al., "High-efficiency GaInP/GaAs/GaInNAs solar cells grown by combined MBE-MOCVD technique" Prog. Photovolt. Res. Appl., vol. 24, no. 7, pp. 914-919, Jul. 2016.

[3] Y. Zhang et al., "GaInP/GaInAs/GaInNAs/Ge Four-Junction Solar Cell Grown by Metal Organic Chemical Vapor Deposition with High Efficiency" Chin. Phys. Lett., vol. 33, no. 10, p. $108801,2016$.

[4] M. Ochoa et al., "Advances Towards 4J Lattice-Matched including Dilute Nitride Subcell for Terrestrial and Space Applications" in 2016 IEEE 43rd Photovoltaic Specialist Conference (PVSC), 2016, p. n/a.

[5] M. Ochoa et al., "Modelling of lattice matched dilute nitride 4junction concentrator solar cells on Ge substrates" AIP Conf. Proc., vol. 1766, no. 1, 2016.

[6] E. Centurioni, "Generalized matrix method for calculation of internal light energy flux in mixed coherent and incoherent multilayers" Appl. Opt., vol. 44, no. 35, pp. 7532-7539, Dec. 2005. 
[7] E. Barrigón Montañés, "Development of GaInP/GaInAs/Ge triple-junction solar cells for CPV applications" Phd, E.T.S.I. Telecomunicación (UPM), 2014

[8] J.-C. Harmand et al., "GaNAsSb: how does it compare with other dilute III-V-nitride alloys?" Semicond. Sci. Technol., vol. 17, no. 8, p. 778, 2002.

[9] R. Kudrawiec et al., "Photoluminescence from as-grown and annealed $\mathrm{GaN}_{0.027} \mathrm{As} \mathrm{s}_{0.863} \mathrm{Sb}_{0.1} / \mathrm{GaAs}$ single quantum wells" $J$. Appl. Phys., vol. 98, no. 6, p. 063527, Sep. 2005.

[10] S. Kurtz, R. King, D. Law, A. Ptak, J. Geisz, and N. Karam, "Effects of in situ annealing on GaInNAs solar cells" in 2013
IEEE 39th Photovoltaic Specialists Conference (PVSC), 2013 pp. 2095-2099.

[11] N. Miyashita, N. Ahsan, and Y. Okada, "Improvement of 1.0 eV GaInNAsSb solar cell performance upon optimal annealing" Phys. Status Solidi A, vol. 214, no. 3, Mar. 2017.

[12] T. Kirchartz, U. Rau, M. Hermle, A. W. Bett, A. Helbig, and J. H. Werner, "Internal voltages in GaInP/GaInAs'Ge multijunction solar cells determined by electroluminescence measurements" Appl. Phys. Lett., vol. 92, no. 12, p. 123502, Mar. 2008. 